\title{
Differential expression of methyltransferase METTL1 associates with death in a cohort of patients with triple negative breast cancer.
}

\author{
Shahan Mamoor ${ }^{1}$ \\ 1Thomas Jefferson School of Law \\ San Diego, CA 92901 \\ mamoorsk@tisl.edu
}

Whole genome profiling data of 226 patients with triple negative breast cancer using a published dataset (1) was stratified based on survival. When comparing the transcriptomes of 87 patients that expired versus 123 patients that survived, the methyltransferase like 1 METTL1 was the third most differentially expressed gene in the entire transcriptome. METTL1 mediates 7-methylguanosine modification of microRNAs and tRNAs $(2,3,4,5,6)$. Increased expression of METTL1 is an indication that the disease course will likely result in fatality in patients with triple negative breast cancer and should be treated accordingly.

Keywords: triple negative breast cancer, differential gene expression analysis, METTL1, systems oncology, comparative transcriptome profiling, targeted cancer therapy 


\section{Introduction}

Breast cancer is a leading cause of cancer death for woman in the United States (7). Breast cancer can be classified by the type of cell from which it originates, such a basal or luminal-type (8), or by the type of receptors it expresses, such as HER2+ for cancers expressing the human epidermal growth factor receptor and ER+ for cancers expressing the estrogen receptor (8). Triple negative breast cancer, or TNBC by definition lacks expression of the estrogen receptor, the progesterone receptor, and does not overexpress the human epidermal growth factor receptor (9). Antibodies targeting HER2+ (trastuzumab, or Herceptin) and endocrine therapies such as tamoxifen are standard of care therapies for woman diagnosed with breast cancers $(10,11)$. Women diagnosed with TNBC will not respond to these therapies like women with other breast cancer types and there is no accepted standard of care for women with TNBC (9). Platinum chemotherapy like carboplatin in conjunction with gemcitabine has been demonstrated to provide a $26-34 \%$ response rate in $\operatorname{TNBC}(12,13,14$, 15). More recently, the poly ADP-ribose polymerase (PARP) inhibitor olaparib, approved for women with BRCA1 mutations, has been used in addition to standard chemotherapy regimens like paclitaxel (anthracyclines) for women with TNBC that harbor a BRCA1 lesion (16), and inaparib has been studies as an adjunct to gemcitabine-carboplatin (17), but neither are specifically approved for women with TNBC. Nearly all of the women diagnosed with TNBC will die of the disease. There is a dire need for targeted therapies for women with TNBC.

Women with triple negative breast cancer, in addition to lacking the standard of care that women with other breast cancers (like HER2+ and ER+) possess, also face a more aggressive disease course. While the median time to death in women with all other types of breast cancer is 6 years, the median time to death in woman with TNBC is 4.2 years (9). Women with TNBC have a $42.2 \%$ mortality rate, while women with other breast cancers have a $28 \%$ mortality rate (9). Metastasis, or the spread of cancer to distant organ sites such as the brain, the bones and the lungs is also more severe in women with TNBC (9). $33.9 \%$ of women 
with TNBC will have a metastatic recurrence of their disease while this rate for women with other breast cancers is $20.4 \%$ (9). While the mean time to distant recurrence for women with other breast cancers is 5 years, recurrence to a distant site will occur in women with TNBC in nearly half of this time -2.6 years (9). While local recurrence, or return of the cancer at the breast occurs at a similar rate in women with TNBC and other breast cancer types (13\% versus $12 \%)$, this recurrence takes place in 2.8 years in women with TNBC versus 4.2 years in women with other breast cancer types (9).

Cancers, in general, have traditionally been treated with surgery to remove any operable cancerous tissue, and treated with chemotherapy and radiation when indicated.

Chemotherapy and radiation therapy are both based on the principle that cells that divide at a faster rate, like cancer cells, will succumb faster to the uptake of what are essentially poisons $(18,19)$. This is the underlying basis to loss of hair and Gl distress in patients undergoing many types of chemotherapy treatment, as hair follicles and cells of the Gl tract divide at a faster rate than other cell types in the body. Imatinib, or Gleevec, is a model drug for a paradigm known as targeted therapy $(20,21)$. In chronic myelogenous leukemias $(\mathrm{CML})$ that are positive for the Philadelphia chromosome, there is an underlying gene fusion known as BCR-ABL that was harnessed as a means to develop a targeted therapy that would specifically affect BCR-ABL+ CML cells rather than all cell types that uptake the drug (22). While resistance to imatinib can develop (23), therapies that target compensatory mutations in patients treated with and resistant to imatinib such as nilotinib and dasitinib have been subsequently developed and used successfully to treat imatinib resistant $B C R-A B L+C M L(24,25)$. A seminal drug trial, IRIS, demonstrated a 19-month cytogenetic response at $87.1 \%$ for patients treated with imatinib versus $34.7 \%$ for patients treated with the previous standard of care, interferon alfa plus the chemotherapy cytarabine (26). At 12 months, the disease had not progressed in $79.9 \%$ of patients treated with interferon plus cytarabine versus $96.6 \%$ in patients treated with imatinib (26). Overall, hematologic response was demonstrated at $95.3 \%$ versus $55.5 \%$ in 
patients with interferon and cytarabine. Median time to response was 1 month in patients treated with imatinib versus 2.5 months in patients treated with cytarabine and interferon (26). Moreover, as might be assumed by the targeted nature of this therapy, imatinib was (overall) significantly more tolerable. While $34.5 \%$ of patients treated with imatinib were fatigued, $65.5 \%$ of patients treated with interferon plus cytarabine were fatigued (26). Grade 3 or 4 adverse events are the worst type of events with respect to severity (26). While $1.1 \%$ of patients treated with imatinib were experienced Grade 3 or 4 fatigue, $24.4 \%$ of patients treated with interferon plus cytarabine experienced Grade 3 or 4 fatigue (26). $12.8 \%$ patients treated with interferon plus cytarabine experienced Grade 3 or 4 depression, while $0.4 \%$ percent of patients treated with imatinib experienced Grade 3 or 4 depression (26). Neutropenia is a hematologic disturbance that can be dangerous if resulting in infection that complicates the course of treatment $(27,28)$. While $25 \%$ of patients treated with interferon plus cytarabine were experienced grade 3 or 4 neutropenia, $14.3 \%$ of patients treated with imatinib experienced grade 3 or 4 neutropenia (26). Not only was imatinib as a single agent superior to the existing standard of care, it was better tolerated. Not only do women with TNBC lack a standard of treatment, there are zero FDA-approved targeted therapies for triple negative breast cancer.

Systems analyses, such as transcriptome and genome-wide analyses do not assume specific targets or cancer vulnerabilities, and instead ask what are the most significant changes or differences between a cancer and a healthy tissue, or between cancer patients who have survived versus cancer patients who have succumbed to the disease (29-38). Here, we performed global differential comparative gene expression analysis using a public dataset of whole transcriptome data from a cohort of 226 African American women with TNBC (1). When stratifying patients based on survival, we found that the tRNA and miRNA N-7methylguanosine transferase METTL1 was the third most significantly differentially expressed gene transcriptome-wide. Women who expired from TNBC expressed METTL1 at significantly 
higher levels than women who had survived the disease. METTL1, as an enzyme with a discrete and targetable catalytic site $(39,40,41)$, demonstrates promise as a therapeutic target in women with TNBC, and its expression at the RNA level is a prognostic indicator of poorer disease outcome and likely fatality.

\section{Methods}

Dataset GSE142102 using platform GPL17692 was used for this analysis, performed using GEO2R. The authors of the study from which this dataset was obtained (Purrington, KS; Wayne State University; NCBI Geo DataSets; unpublished) used Affymetrix Human Gene 2.1 ST Array microarray transcriptome technology to perform whole genome profiling of fresh frozen paraffin embedded (FFPE) tumors from 226 African American women diagnosed with triple negative breast cancer, or TNBC. For the global differential gene expression analysis conducted here, $p$-value adjustment was set to Benjamini and Hochberg; log transformation of data was set to "Auto-detect", and the submitter supplied category of platform annotation was used. Statistical significance of differential expression was determined by GEO2R. Statistical significance to compare the mean value of RNA expression between dead and alive groups was performed using PRISM (PRISM 8.1.2) to conduct unpaired t-tests with Welch's correction.

\section{Results}

We performed differential gene expression analysis of a cohort of 226 African American women diagnosed with triple negative breast cancer, or TNBC (1). Of the 226 patients, 87 had expired from the disease while 123 were still surviving; the survival status of 16 patients out of 226 was not designated and as such their expression data was not included in this analysis. We stratified the cohort based on survival and compared the transcriptomes of the 87 women who had succumbed to the disease to the transcriptomes of the 123 women who had survived, to determine in an unbiased, global fashion which genes were most differently expressed between the two groups. This analysis of TNBC patients revealed that out of 53617 
total transcripts detected by microarray, METTL1 was the third most differentially expressed gene when comparing survivors to those who had expired from the disease (Table 1). METTL1 is 7-methylguanosine $\left(m^{7} \mathrm{G}\right)$ methyltransferase for transfer RNAs (tRNAs) that function in translation $(2,3,5)$ and was recently identified as a $m^{7} G$ methyltransferase for certain microRNAs as well $(4,5)$. In conjunction with WDR4, it modifies tRNAs at position 46 from yeast to humans with the $\mathrm{m}^{7} \mathrm{G}$ mark (3).

The breast tumors of the 127 surviving patients in this TNBC cohort expressed significantly lower levels of METTL1 RNA (Figure 1A). In the 87 TNBC patients who had expired, METTL1 RNA was expressed at significantly higher levels than in patients who had survived (Figure 1B). The individual expression of METTL1 RNA by patient varied, and some TNBC survivors expressed higher levels of METTL1 than some TNBC patients who had expired (Figure 1C). Similarly, some TNBC patients who had expired had lower levels of METTL1 RNA than some TNBC patients who had survived (Figure 1C). However, METTL1 was the third most differentially expressed gene between when comparing the tumors of TNBC patients surviving or expiring, and on average, the breast tumors of TNBC patients who died expressed significantly higher levels of METTL1 RNA.

To attempt to correlate METTL1 expression level with survival or fatality, we directly assessed the RNA expression value of the transcript of METTL1 enzyme in the tumors of women with TNBC that expressed METTL1 at the highest levels. In total, 22 women expressed METTL1 transcript at a value greater than 1. 17 of these women were dead, while only 4 had survived (Figure $2 \mathrm{C}$ ); this approached statistical significance (Figure $2 \mathrm{~A} ; p=0.0574)$. When performing the same analysis with women whose tumors expressed METTL1 at levels greater than 1.5, the correlation between higher METTL1 expression and risk of death was statistically significant (Figure 2B; $p=0.0389$ ). To assess whether lower expression of METTL1 would correlate survival, we performed the same analysis by comparing the values of METTL1 in the 
tumors of women who expressed METTL1 at the lowest levels. 23 women expressed METTL1 transcript at levels less than $-1 ; 15$ out of 23 women whose tumors expressed METTL1 transcript at values less than -1 were alive (Figure 2D). Though higher expression of METTL1 significantly correlated with death (Figure 2B), lower expression of METTL1 was not statistically significantly correlated with survival (Figure 2D). Percentage-wise, the likelihood of survival based on the highest and lowest expression of METTL1 RNA in this cohort, for women with TNBC whose tumors expressed the highest levels of METTL1 was 18.2\% (4/22), and for women with TNBC whose tumors expressed the lowest levels of METTL1 was $65.2 \%$ (15/23).

\section{Discussion}

We performed whole genome profiling of transcriptome data from a public dataset of a cohort of 226 African American women with triple negative breast cancer (1), an aggressive and more fatal type of breast cancer with poorer treatment options than other breast cancers (9). This analysis revealed, when tumor gene expression profiles of the 87 women with TNBC that expired were blindly compared to the tumor gene expression profiles of the 123 women with TNBC that survived, that the tRNA and miRNA $m^{7} G$ methyltransferase METTL1 was the third most differentially expressed gene transcriptome-wide. METTL1 was expressed at significantly higher levels, on average in women with TNBC that expired and the women with highest expressed of METTL1 in this cohort were significantly more likely to expire.

Pandolfini et. al recently identified an miRNA methyltransferase function for METTL1 and found that through its catalytic site was able to exert influence on cell migration (4). Short hairpin knockdown of METTL1 in the A549 lung cancer cell line increased migration capacity in an in vitro migration assay, but did not have an influence on cell proliferation in vitro nor did it have an affect on overall mRNA translation (4). Thus, the authors concluded that METTL1 appeared to have an effect on migration through its catalytic site through its $\mathrm{m}^{7} \mathrm{G}$ 
methyltransferase function since a catalytically inactivate version of the enzyme did not have a similar influence on cell migration (4).

In mouse embryonic stem cells, METTL1 is required for self-renewal and knockdown of METTL1 results in defects in colony size and colony number (6). mESC with METTL1 shRNA knockdown display decreased expression of ectoderm germline markers (Pax6, Otx2, Fgf5, Sox1) with concurrent increased expression of endoderm (Eomes, T, Mixl1, Snail) and mesoderm (Gata4, Gata6, Sox17, and Foxa2) markers (6). Knockout of METTL1 perturbs differentiation of mESC towards the neural lineage, and the authors showed that the CDS and UTR of genes required for the neural differentiation program were significantly longer than a random collection of genes, providing evidence that inhibition of the $m^{7} G$ methyltransferase activity was specifically responsible for this defect (6). Together, these studies demonstrated that by its $\mathrm{m}^{7} \mathrm{G}$ methyltransferase catalytic activity, METTL1 is required for the self-renewal capacity of mouse embryonic stem cells, plays an important role in the differentiation of pluripotent mESC cells into the neural lineage, and can exert an influence on cell migration in at least one type of human lung cancer cell line (6). Significantly, one recent study has reported correlation of METTL1 over-expression with poorer survival in hepatocellular carcinoma (43), and another unpublished study has suggested a role for METTL1 in glioblastoma multiforme (44).

The data from this global comparative transcriptome analysis from 266 patients demonstrates that METTL1 is among the most differentially expressed genes in a cohort of patients with triple negative breast cancer (TNBC) when their transcriptomes are stratified based on survival, that it is expressed at significantly higher levels in patients dying from TNBC versus those surviving the disease, and that those with the highest expression of TNBC are at significantly higher risk of dying from the disease. There are have been no studies demonstrating a role for METTL1 in breast cancer, in general or in TNBC. The fact that 
METTL1 is expressed at relatively higher levels in patients with TNBC more likely to die from the disease, that it plays a role in the self-renewal capacity of murine embryonic stem cells (6), and that it exerts control over cell migration in at least one human lung cancer cell line (4) together suggest that METTL1 is a promising therapeutic target in TNBC. The expression of TNBC should be validated at the protein level and in larger and different cohorts than the cohort of African American woman studied here. Knockdown of METTL1 in TNBC cancer cell lines should be studied for effects on proliferation, migration and anchorage-independent growth capacity in vitro, and xenograft models of TNBC (45) should be assessed for effects on metastasis and survival in vivo.

Efforts should be prioritized towards assessing whether patients with TNBC have specific dependencies on METTL1, whether its catalytic activity is required for such a dependency. This could involve chemical synthesis of METTL1 small molecule inhibitors, generation of tRNA decoy sequences and/or structural analogs with high affinity for METTL1 to divert its catalytic activity in a manner similar to NRTI chain terminators and the HIV-1 reverse transcriptase (46). As Protein Kinase B (PKB) and Ribosomal S6 Kinase (RSK) phosphorylation inactivate METTL1 activity (47), these could also be targeted to inhibit METTL1, but would likely result in pleiotropic effects and compromise the specific, targeted nature of therapeutic target discovery by systems-wide analysis of tumor transcription. Inactivation of METTL1 by inhibition of its binding partner WDR4 $(3,4,6,47)$ or the binding interface between METTL1 and WDR4 could be another means of targeting METTL1 enzymatic activity independent of its catalytic site.

METTL1 is the third most differentially expressed gene in a cohort of patients with triple negative breast cancer when comparing the transcriptomes of TNBC patients that survive with those that expire from the disease. METTL1 RNA is expressed at significantly higher levels in patients who die from a type of breast cancer with no available targeted therapies and with 
poorer prognostic outcomes (9). Women with TNBC have a shorter time to local recurrence (the amount of time it takes for the disease to return to the breast), percent of patients experiencing metastatic recurrence, or spread of cancer to a foreign site such as the brain, bones or lung after treatment, a shorter time to metastatic recurrence, a higher overall percent fatality, and on average a shorter time to death (9). Our findings demonstrate that METTL1 RNA expression is a prognostic indicator of death in women with TNBC whose tumors express the highest levels of METTL1. METTL1 should be prioritized as a therapeutic target in triple negative breast cancer. 


\section{References}

1. Purrington, KS; Wayne State University; GEO Data Sets GSE142102.

2. Bahr, A., Hankeln, T., Fiedler, T., Hegemann, J. and Schmidt, E.R., 1999. Molecular Analysis of METTL1, a Novel Human Methyltransferase-like Gene with a High Degree of Phylogenetic Conservation. Genomics, 57(3), pp.424-428.

3. Alexandrov, A., Martzen, M.R. and Phizicky, E.M., 2002. Two proteins that form a complex are required for 7-methylguanosine modification of yeast tRNA. Rna, 8(10), pp.1253-1266.

4. Pandolfini, L., Barbieri, I., Bannister, A.J., Hendrick, A., Andrews, B., Webster, N., Murat, P., Mach, P., Brandi, R., Robson, S.C. and Migliori, V., 2019. METTL1 promotes let-7 microRNA processing via m7G methylation. Molecular cell, 74(6), pp.1278-1290.

5. Zhang, L.S., Liu, C., Ma, H., Dai, Q., Sun, H.L., Luo, G., Zhang, Z., Zhang, L., Hu, L., Dong, $X$. and He, C., 2019. Transcriptome-wide mapping of internal N7-methylguanosine methylome in mammalian mRNA. Molecular cell, 74(6), pp.1304-1316.

6. Lin, S., Liu, Q., Lelyveld, V.S., Choe, J., Szostak, J.W. and Gregory, R.I., 2018. Mettl1/Wdr4mediated $\mathrm{m} 7 \mathrm{G}$ tRNA methylome is required for normal mRNA translation and embryonic stem cell self-renewal and differentiation. Molecular cell, 71(2), pp.244-255.

7. DeSantis, C.E., Fedewa, S.A., Goding Sauer, A., Kramer, J.L., Smith, R.A. and Jemal, A., 2016. Breast cancer statistics, 2015: Convergence of incidence rates between black and white women. CA: A Cancer Journal for Clinicians, 66(1), pp.31-42.

8. Kennecke, H., Yerushalmi, R., Woods, R., Cheang, M.C.U., Voduc, D., Speers, C.H., Nielsen, T.O. and Gelmon, K., 2010. Metastatic behavior of breast cancer subtypes. Journal of clinical oncology, 28(20), pp.3271-3277.

9. Dent, R., Trudeau, M., Pritchard, K.I., Hanna, W.M., Kahn, H.K., Sawka, C.A., Lickley, L.A., Rawlinson, E., Sun, P. and Narod, S.A., 2007. Triple-negative breast cancer: clinical features and patterns of recurrence. Clinical cancer research, 13(15), pp.4429-4434.

10. Piccart-Gebhart, M.J., Procter, M., Leyland-Jones, B., Goldhirsch, A., Untch, M., Smith, I., Gianni, L., Baselga, J., Bell, R., Jackisch, C. and Cameron, D., 2005. Trastuzumab after adjuvant chemotherapy in HER2-positive breast cancer. New England Journal of Medicine, 353(16), pp.1659-1672.

11. Romond, E.H., Perez, E.A., Bryant, J., Suman, V.J., Geyer Jr, C.E., Davidson, N.E., TanChiu, E., Martino, S., Paik, S., Kaufman, P.A. and Swain, S.M., 2005. Trastuzumab plus adjuvant chemotherapy for operable HER2-positive breast cancer. New England Journal of Medicine, 353(16), pp.1673-1684.

12. Chew, H.K., Doroshow, J.H., Frankel, P., Margolin, K.A., Somlo, G., Lenz, H.J., Gordon, M., Zhang, W., Yang, D., Russell, C. and Spicer, D., 2009. Phase II studies of gemcitabine and cisplatin in heavily and minimally pretreated metastatic breast cancer. Journal of Clinical Oncology, 27(13), p.2163.

13. Koshy, N., Quispe, D., Shi, R., Mansour, R. and Burton, G.V., 2010. Cisplatin-gemcitabine therapy in metastatic breast cancer: Improved outcome in triple negative breast cancer patients compared to non-triple negative patients. The Breast, 19(3), pp.246-248.

14. Zhang, J., Wang, Z., Hu, X., Wang, B., Wang, L., Yang, W., Liu, Y., Liu, G., Di, G., Hu, Z. and Wu, J., 2015. Cisplatin and gemcitabine as the first line therapy in metastatic triple negative breast cancer. International journal of cancer, 136(1), pp.204-211.

15. Reeder-Hayes, K.E., Carey, L.A. and Sikov, W.M., 2011. Clinical trials in triple negative breast cancer. Breast disease, 32(1-2), pp.123-136.

16. Gelmon, K.A., Tischkowitz, M., Mackay, H., Swenerton, K., Robidoux, A., Tonkin, K., Hirte, H., Huntsman, D., Clemons, M., Gilks, B. and Yerushalmi, R., 2011. Olaparib in patients with recurrent high-grade serous or poorly differentiated ovarian carcinoma or triplenegative breast cancer: a phase 2, multicentre, open-label, non-randomised study. The lancet oncology, 12(9), pp.852-861. 
17. O'Shaughnessy, J., Osborne, C., Pippen, J.E., Yoffe, M., Patt, D., Rocha, C., Koo, I.C., Sherman, B.M. and Bradley, C., 2011. Iniparib plus chemotherapy in metastatic triplenegative breast cancer. New England Journal of Medicine, 364(3), pp.205-214.

18. DeVita, V.T. and Chu, E., 2008. A history of cancer chemotherapy. Cancer research, 68(21), pp.8643-8653.

19. Chabner, B.A. and Roberts Jr, T.G., 2005. Chemotherapy and the war on cancer. Nature Reviews Cancer, 5(1), p.65.

20. Nahta, R., Yu, D., Hung, M.C., Hortobagyi, G.N. and Esteva, F.J., 2006. Mechanisms of disease: understanding resistance to HER2-targeted therapy in human breast cancer. Nature Reviews Clinical Oncology, 3(5), p.269.

21. Savage, D.G. and Antman, K.H., 2002. Imatinib mesylate-a new oral targeted therapy. New England Journal of Medicine, 346(9), pp.683-693.

22. Capdeville, R., Buchdunger, E., Zimmermann, J. and Matter, A., 2002. Glivec (STI571, imatinib), a rationally developed, targeted anticancer drug. Nature reviews Drug discovery, 1(7), p.493.

23. Shah, N.P., Nicoll, J.M., Nagar, B., Gorre, M.E., Paquette, R.L., Kuriyan, J. and Sawyers, C.L., 2002. Multiple BCR-ABL kinase domain mutations confer polyclonal resistance to the tyrosine kinase inhibitor imatinib (STI571) in chronic phase and blast crisis chronic myeloid leukemia. Cancer cell, 2(2), pp.117-125.

24. Kantarjian, H., Giles, F., Wunderle, L., Bhalla, K., O'Brien, S., Wassmann, B., Tanaka, C., Manley, P., Rae, P., Mietlowski, W. and Bochinski, K., 2006. Nilotinib in imatinib-resistant CML and Philadelphia chromosome-positive ALL. New England Journal of Medicine, 354(24), pp.2542-2551.

25. Talpaz, M., Shah, N.P., Kantarjian, H., Donato, N., Nicoll, J., Paquette, R., Cortes, J., O'Brien, S., Nicaise, C., Bleickardt, E. and Blackwood-Chirchir, M.A., 2006. Dasatinib in imatinib-resistant Philadelphia chromosome-positive leukemias. New England Journal of Medicine, 354(24), pp.2531-2541.

26. O'Brien, S.G., Guilhot, F., Larson, R.A., Gathmann, I., Baccarani, M., Cervantes, F., Cornelissen, J.J., Fischer, T., Hochhaus, A., Hughes, T. and Lechner, K., 2003. Imatinib compared with interferon and low-dose cytarabine for newly diagnosed chronic-phase chronic myeloid leukemia. New England Journal of Medicine, 348(11), pp.994-1004.

27. Pizzo, P.A., 1993. Management of fever in patients with cancer and treatment-induced neutropenia. New England Journal of Medicine, 328(18), pp.1323-1332.

28. Kuderer, N.M., Dale, D.C., Crawford, J., Cosler, L.E. and Lyman, G.H., 2006. Mortality, morbidity, and cost associated with febrile neutropenia in adult cancer patients. Cancer, 106(10), pp.2258-2266.

29. Yarden, Y. and Pines, G., 2012. The ERBB network: at last, cancer therapy meets systems biology. Nature Reviews Cancer, 12(8), p.553.

30. Fitzgerald, J.B., Schoeberl, B., Nielsen, U.B. and Sorger, P.K., 2006. Systems biology and combination therapy in the quest for clinical efficacy. Nature chemical biology, 2(9), p.458.

31. Chin, L., Andersen, J.N. and Futreal, P.A., 2011. Cancer genomics: from discovery science to personalized medicine. Nature medicine, 17(3), p.297.

32. Liang, P. and Pardee, A.B., 2003. Analysing differential gene expression in cancer. Nature Reviews Cancer, 3(11), p.869.

33. Ramaswamy, S. and Golub, T.R., 2002. DNA microarrays in clinical oncology. Journal of clinical oncology, 20(7), pp.1932-1941.

34. Antoniou, A.C., Wang, X., Fredericksen, Z.S., McGuffog, L., Tarrell, R., Sinilnikova, O.M., Healey, S., Morrison, J., Kartsonaki, C., Lesnick, T. and Ghoussaini, M., 2010. A locus on $19 \mathrm{p} 13$ modifies risk of breast cancer in BRCA1 mutation carriers and is associated with hormone receptor-negative breast cancer in the general population. Nature genetics, $42(10)$, p.885. 
35. Cieślik, M. and Chinnaiyan, A.M., 2018. Cancer transcriptome profiling at the juncture of clinical translation. Nature Reviews Genetics, 19(2), pp.93-109.

36. Liotta, L. and Petricoin, E., 2000. Molecular profiling of human cancer. Nature Reviews Genetics, 1(1), p.48.

37. Liu, Y.R., Jiang, Y.Z., Xu, X.E., Hu, X., Yu, K.D. and Shao, Z.M., 2016. Comprehensive transcriptome profiling reveals multigene signatures in triple-negative breast cancer. Clinical cancer research, 22(7), pp.1653-1662.

38. Cabaye, A., Nguyen, K.T., Liu, L., Pande, V. and Schapira, M., 2015. Structural diversity of the epigenetics pocketome. Proteins: Structure, Function, and Bioinformatics, 83(7), pp. 1316-1326.

39. Leulliot, N., Chaillet, M., Durand, D., Ulryck, N., Blondeau, K. and van Tilbeurgh, H., 2008. Structure of the yeast tRNA m7G methylation complex. Structure, 16(1), pp.52-61.

40. Schapira, M., 2015. Structural chemistry of human RNA methyltransferases. ACS chemical biology, 11(3), pp.575-582.

41. Lin, S., Liu, Q., Lelyveld, V.S., Choe, J., Szostak, J.W. and Gregory, R.I., 2018. Mettl1/Wdr4mediated $\mathrm{m} 7 \mathrm{G}$ tRNA methylome is required for normal mRNA translation and embryonic stem cell self-renewal and differentiation. Molecular cell, 71(2), pp.244-255.

42. Tian, Q.H., Zhang, M.F., Zeng, J.S., Luo, R.G., Wen, Y., Chen, J., Gan, L.G. and Xiong, J.P., 2019. METTL1 overexpression is correlated with poor prognosis and promotes hepatocellular carcinoma via PTEN. Journal of Molecular Medicine, 97(11), pp.1535-1545.

43. Seaman, J. and Endres, L., 2017. Putative Role for METTL1 in Human Gliobastoma.

44. Chen, X., lliopoulos, D., Zhang, Q., Tang, Q., Greenblatt, M.B., Hatziapostolou, M., Lim, E., Tam, W.L., Ni, M., Chen, Y. and Mai, J., 2014. XBP1 promotes triple-negative breast cancer by controlling the HIF1a pathway. Nature, 508(7494), p.103.

45. Cihlar, T. and Ray, A.S., 2010. Nucleoside and nucleotide HIV reverse transcriptase inhibitors: 25 years after zidovudine. Antiviral research, 85(1), pp.39-58.

46. Cartlidge, R.A., Knebel, A., Peggie, M., Alexandrov, A., Phizicky, E.M. and Cohen, P., 2005. The tRNA methylase METTL1 is phosphorylated and inactivated by PKB and RSK in vitro and in cells. The EMBO journal, 24(9), pp.1696-1705. 


\section{METTL1}

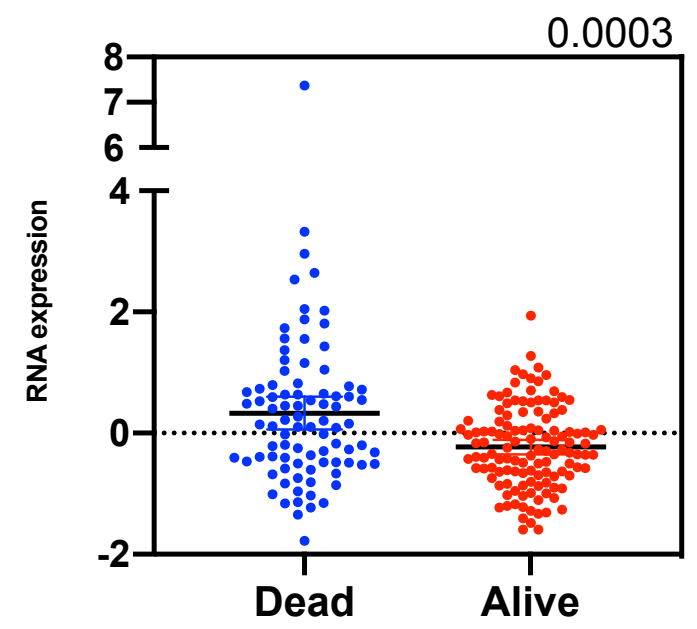

A

B
METTL1

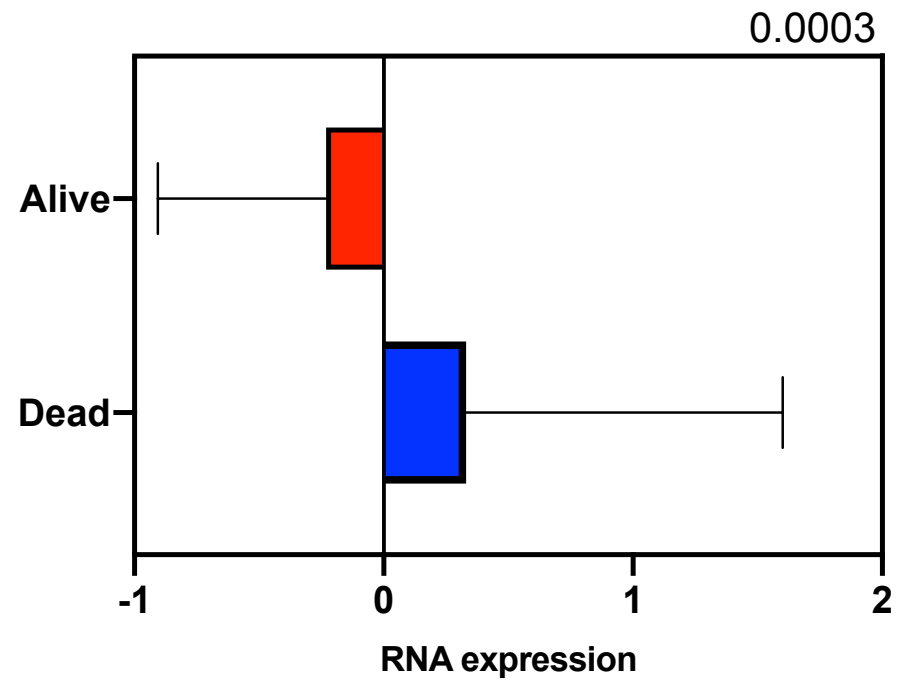

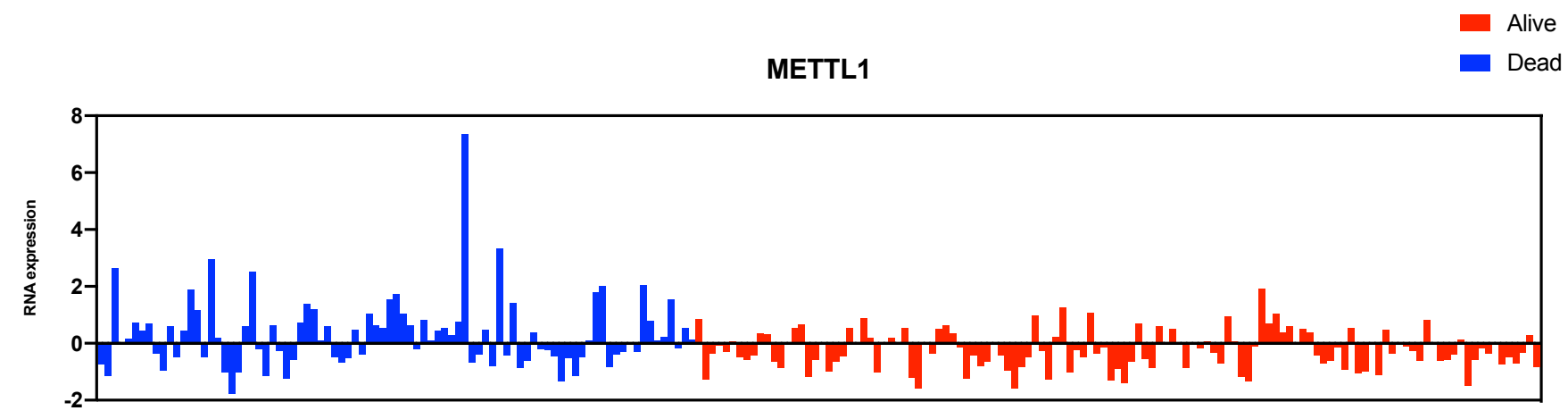

C

Figure 1: METTL1 transcript is the third most differentially expressed gene in a cohort of 266 women with triple negative breast cancer (TNBC) when comparing survivors to the deceased.

Figure 1A: The RNA expression value of METTL1 in patients dead and alive, with the mean value designated by the horizontal black bar. Statistical significance as designated by the $p-$ value above the graph, was calculated using an unpaired t-test with Welch's correction.

Figure 1B: Mean expression of the METTL1 transcript with standard deviation of the mean designated by the error bars. Statistical significance was calculated using an unpaired t-test with Welch's correction, and shown above the graph.

Figure 1C: The RNA expression value of METTL1 in each individual patient is graphically represented. 
METTL1 expression > 1

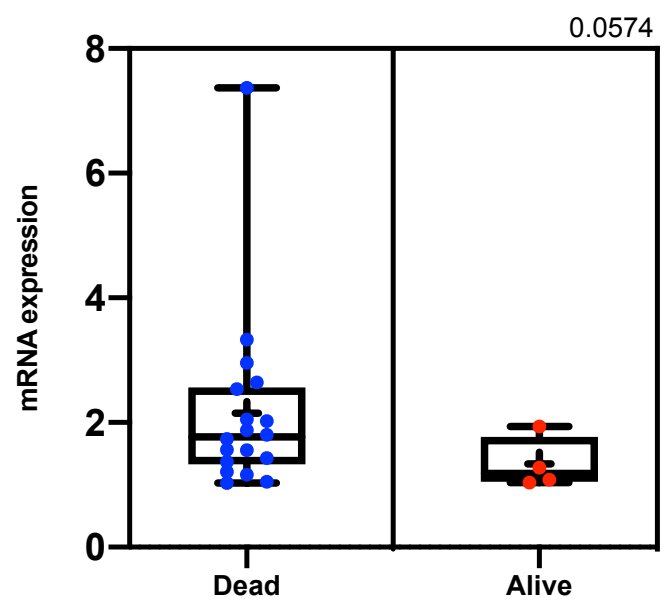

METTL1 expression > 1.5

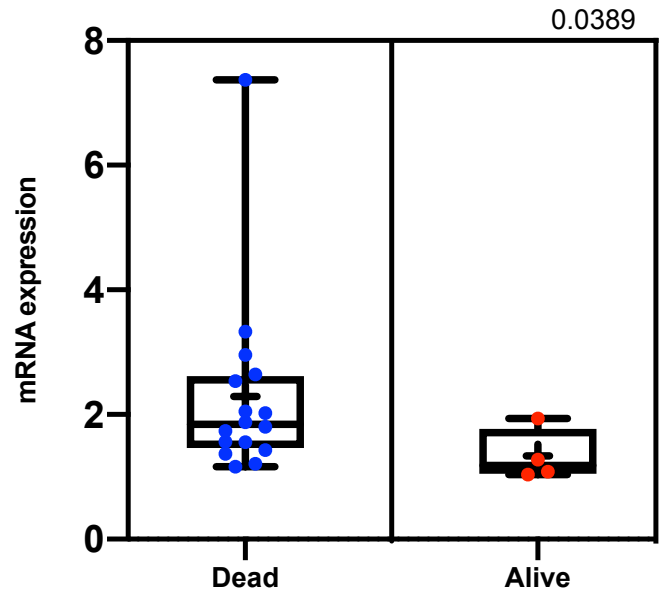

A

B

METTL1 expression > 1

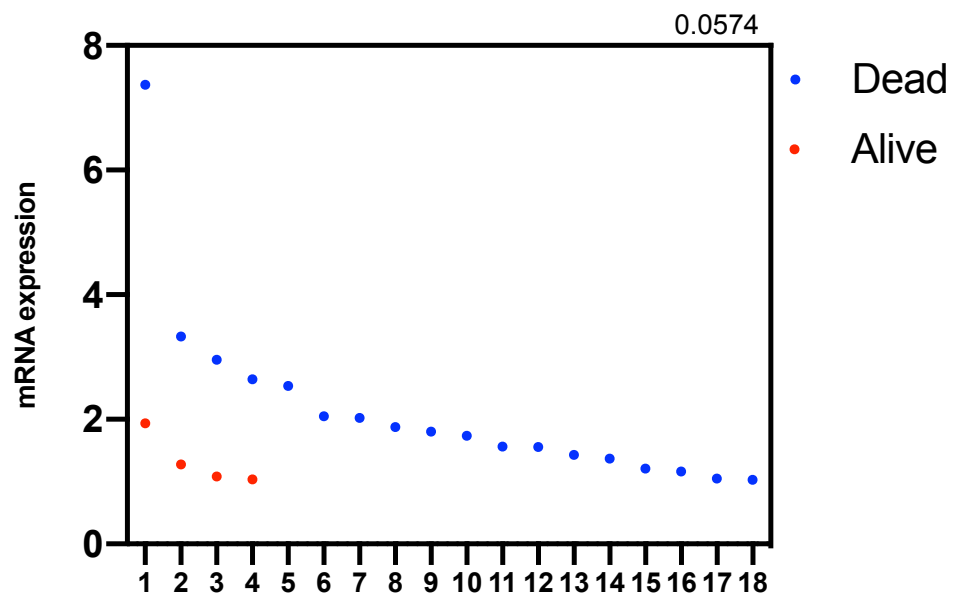

METTL1 expression $<-1$

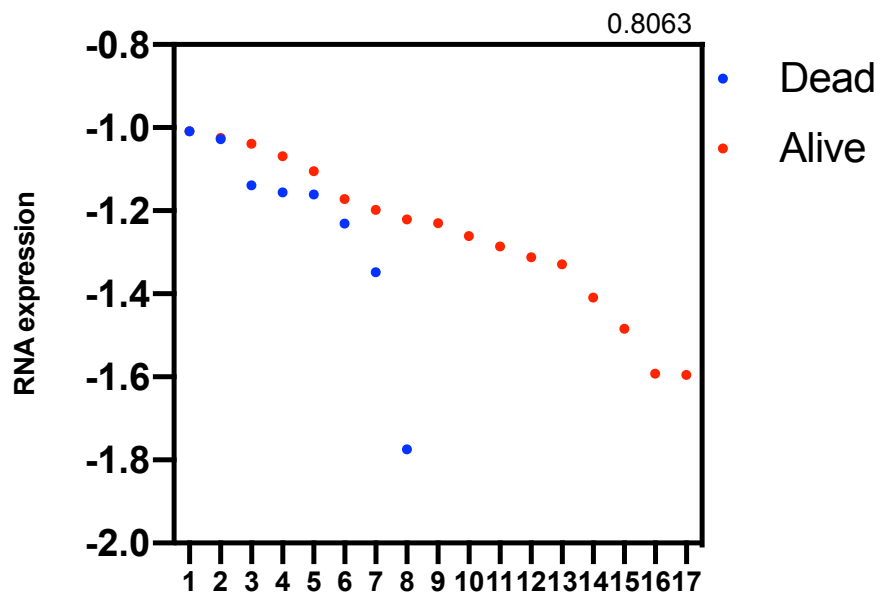

C

D

Figure 2: Higher expression of METTL1 transcript correlates with significantly increased risk of death in TNBC.

Figure 2A and 2B: Patients expressing METTL1 transcript at levels higher than "1" (left, 2A) or "1.5" (right, 2B) were stratified based on survival and an unpaired t-test with Welch's correction was performed ( $p$-value indicated on each graph) to determine statistical significance of difference between mean values of each group.

Figure 2C and 2D: Patients expressing METTL1 transcript at levels higher than "1" (left, 2C) or lower than "-1" (right, 2D) were stratified based on survival and an unpaired t-test with Welch's correction was performed ( $p$-value indicated on each graph) to determine statistical significance of difference between mean values of each group. 
Top 250 Differentially Expressed Genes in Cohort of 266 Women with Triple Negative Breast Cancer

\begin{tabular}{|l|r|r|r|r|r|}
\hline Gene & \multicolumn{1}{l}{ Rank } & \multicolumn{1}{l}{$\boldsymbol{p}$-value } & B & \multicolumn{1}{l|}{ I } \\
\hline Mettl1 & 3 & 0.00006057 & -2.07 & -4.01 & ENST00000324871 \\
\hline
\end{tabular}

Table 1: METTL1 transcript is the third most differentially expressed gene in a cohort of 266 women with triple negative breast cancer (TNBC): data from GEO2R whole transcriptome differential gene expression analysis.

Data provided here includes rank of differential expression relative to the transcriptome profiled, raw $p$-value for statistical significance of differential expression of the gene, log-odds that the gene is differentially expressed (B-statistic), and a moderated t statistic generated by comparison of the two groups (dead and alive). 